\title{
Aprender Bioética desde la Re-existencia
}

\author{
MUÑOS, Favio Rivas ${ }^{1}$
}

\section{RESUMEN}

Es aceptado que movimientos sociales de diferente tipo jugaron algún papel de importancia en el surgimiento de la bioética en las últimas décadas del siglo XX, junto con los avances biomédicos y la experimentación con seres humanos. Sin embargo, hoy dichos movimientos yacen en el olvido dado el énfasis de esta inter-disciplina científica en lo estrictamente biomédico y su práctica encerrada en comités éticos de asistencia e investigación donde se adelanta, a su vez, un ejercicio de democracia liberal, representativa o comisionada, el paradigma de democracia en el llamado occidente al Norte y al Sur. Latinoamérica, en particular, ha sido y sigue siendo un continente rico en movimientos sociales, según informe del sistema de Naciones Unidas. Entre ellos, los llamados Movimientos contra-hegemónicos son movimientos de resistencia en diferentes partes del mundo que ponen de manifiesto elementos de importancia hacia la construcción de una bioética más acorde con las realidades del Sur global. Como movimientos de resistencia a esa centralidad en el negocio, propia de esta época, los seres humanos a su interior han ido haciéndose a otras formas de vida, es decir, de re-existencia, que rebelan opciones para la supervivencia de la especie humana en un mundo que arrasa y depreda para sólo favorecer la rentabilidad de inversiones a escala global.

Bioética. Bioética De Intervención. Movimientos Sociales Contra-Hegemónicos. América Latina.

\section{Aprender Bioética a Partir da Re-existência}

\section{RESUMO}

Aceita-se que movimentos sociais de diferentes tipos tenham desempenhado papel importante no surgimento da bioética nas últimas décadas do século $\mathrm{XX}$, juntamente com avanços biomédicos e experimentação com seres humanos. No entanto, hoje esses movimentos estão no esquecimento, dada a ênfase dessa interdisciplina científica no estritamente biomédico e sua prática encerrada em comitês éticos de assistência e pesquisa, onde, por sua vez, um exercício liberal, representativo ou comissário, o paradigma da democracia no chamado oeste para o norte e sul. A América Latina, em particular, tem sido e continua sendo um continente rico em movimentos sociais, de acordo com um relatório do sistema das Nações Unidas. Entre eles, os chamados movimentos contra-

1 Profesor Asociado, Departamento de Salud Pública, Facultad de Medicina, Universidad Nacional de Colombia. Magister Enfermedades Tropicales, Epidemiología y Bioética. PhD Bioética UnB. Email: farivasm@unal.edu.com.

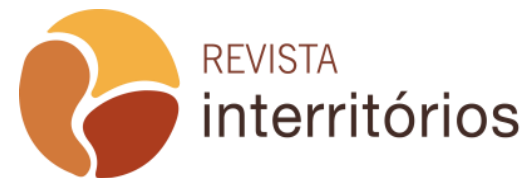


hegemônicos são movimentos de resistência em diferentes partes do mundo que revelam elementos importantes para a construção de uma bioética mais alinhada às realidades do sul global. Como movimentos de resistência a essa centralidade nos negócios, típicos da época, os seres humanos têm criado outras formas de vida, isto é, de re-existência, que revelam opções para a sobrevivência da espécie humana em um mundo que devasta e deprecia apenas para favorecer a lucratividade dos investimentos em escala global.

Bioética. Bioética De Intervenção. Movimentos Sociais Contra-Hegemônicos. América Latina.

\title{
Learn Bio-Ethics Starting from Re-existence
}

\begin{abstract}
It is accepted that social movements of different types have played an important role in the emergence of bioethics in the last decades of the 20th century, together with biomedical progress and experimentation with humans. However, today these movements are in oblivion, given the emphasis of this scientific interdiscipline on strictly biomedical and its practice contained in ethical committees of assistance and research where, in turn, an exercise of liberal, representative or commissioner, the paradigm of democracy in the so-called west to north and south. Latin America in particular has been and continues to be a continent rich in social movements, according to a report by the United Nations system. Among these, the so-called counter-hegemonic movements are resistance movements in different parts of the world that reveal important elements towards the construction of a bioethics more in line with the realities of the global South. As movements of resistance to this centrality in the business world, typical of this period, human beings within them have created other forms of life, that is, of new existence, which reveal options for the survival of the human species in a world that devastates and he was only preying on the profitability of investments on a global scale.
\end{abstract}

Bioethics. Bioethics of Intervention. Counter-Hegemonic Social Movements. Latin America.

\section{Imparare Bio-Ética Partendo da la Re- esistenza}

\section{RIASSUNTO}

È accettato che movimenti sociali di diverso tipo abbiano giocato un ruolo importante nell'emergere della bioetica negli ultimi decenni del $20^{\circ}$ secolo, insieme ai progressi biomedici e alla sperimentazione con gli esseri umani. Tuttavia, oggi questi movimenti si trovano nell'oblio, data l'enfasi di questa interdisciplina scientifica sul rigorosamente biomedico e la sua pratica racchiusa in comitati etici di assistenza e ricerca dove, a loro volta, un esercizio di liberale, rappresentativo o commissario, il paradigma della democrazia nel cosiddetto 
ovest a nord e sud. L'America Latina, in particolare, è stata e continua ad essere un continente ricco di movimenti sociali, secondo un rapporto del sistema delle Nazioni Unite. Tra questi, i cosiddetti movimenti contro-egemonici sono movimenti di resistenza in diverse parti del mondo che rivelano elementi importanti verso la costruzione di una bioetica più in linea con le realtà del Sud globale. Come movimenti di resistenza a questa centralità nel mondo degli affari, tipici di questo periodo, gli esseri umani al loro interno hanno creato altre forme di vita, cioè di nuova esistenza, che rivelano opzioni per la sopravvivenza della specie umana in un mondo che devasta e depredava solo per favorire la redditività degli investimenti su scala globale.

Bioetica. Bioetica Dell'intervento. Movimenti Sociali Contro-Egemonici. America Latina.

\section{Una introducción necesaria}

La idea, con sus categorías, surge de la acción y debe retornar a la acción, so pena de desmedro para el agente.

Proudhon, 1858.

En el espacio académico de los llamados estudios decoloniales se han propuesto cuatro categorías para la colonialidad, entendida como patrón de poder instaurado con la modernidad y que jerarquiza culturas, saberes, experiencias y vidas, el cual termina administrando la vida en aras de proteger y reproducir el capital. Habría una colonialidad del Poder, con una raza superior, el blanco por encima de mestizos, indios y negros; del Saber que solamente valida como verdadero el conocimiento procedente del marco científico-racional europeo/norteamericano, esencialmente blanco y masculino; del Ser que descarta al no civilizado, o bárbaro, por no estar formado en el ámbito científicoracional y; de la Naturaleza o la Vida, soportada en el dualismo naturalezasociedad para la cual la naturaleza, la vida misma, es objeto, aquello donde el conocimiento científico-racional se aplica. (QUIJANO 2003, GROSFOGUEL 2005, WALSH 2012).

En el contexto de la decolonialidad, aquel proceso de resistencia y de transgresión a la colonialidad, sus referentes más lejanos en el tiempo no son precisamente académicos, son vivenciales, históricamente sangrantes, llenos hasta el cansancio de sufrimiento, de lágrimas, pero dejaron evidencia escrita, uno es el texto del inca Waman Poma de Ayala, quien en 1616 escribe al rey de España Nueva crónica y buen gobierno, por un lado, y el otro de Otabbah Cugoano, esclavo africano vendido en el Caribe, quien escribe en 1787 Thoughts and semtiments on the evil of slavery. Ambos, desde el cristianismo, a partir de sus propias experiencias, lo que vieron y vivieron en su época, muestran, entre otras cosas, la doble moral de los colonizadores españoles e ingleses. Para Poma de Ayala, si el gobernante debe ser alguien virtuoso, la realidad muestra 
a las claras que no lo es, pero, por otro lado, refiere con firmeza que se puede ser virtuoso siendo indio. Para Cugoano, no es suficiente la igualdad del negro ante la ley, esa igualdad gobierno-persona debe ser también persona-persona, de lo contrario, de nada sirve. Las dos fueron posturas eminentemente transgresoras del orden impuesto tras la invasión europea en América Latina. Ese patrón jerárquico de poder/saber (modernidad/colonialidad) comienza a cuestionarse muy temprano por los dos subversivos a que se ha hecho referencia. Serían estos los primeros pasos que camina la decolonialidad en esta parte del continente americano. Antes de 1492 había una visión orgánica del mundo donde todo estaba interrelacionado: la naturaleza, el ser humano, el conocimiento. Con la conquista y la colonia, humano y naturaleza son separados y la función del conocimiento es controlar racionalmente el mundo, dominarlo, ponerlo al servicio del humano, entendido como el hombre blanco, racional, heterosexual y europeo. Analizar se constituyó en la clave para conocer la naturaleza a fin de dominarla y ello significó fragmentarla para entenderla, lo cual, más tarde, permitiría recomponerla según un orden lógico-matemático. (CASTRO-GÓMEZ, 2007).

\section{Bioética, Diálogo y Democracia}

La bioética se ha interesado por los asuntos relacionados con la salud, pero hoy está dedicada casi por completo a dar respuesta a los conflictos éticos en el escenario de la atención y de la investigación biomédicas. En un texto ya clásico acerca de promoción de la salud, editado por OPS/OMS en 1996, hay una definición muy interesante, la cual no parece interesar, o quizá se desconozca, a la bioética principialista, pero viene muy bien al presente trabajo, dice que salud es la "magnitud en que un individuo o grupo pueden, por una parte, realizar sus aspiraciones y satisfacer sus necesidades y por otra, cambiar su entorno o afrontarlo" (KICKBUSCH, 1996). Es aquello de realizar aspiraciones, satisfacer necesidades y cambiar o afrontar el entorno, individual o grupalmente, lo relevante. Puede leerse ahí el papel de protagonistas que los seres humanos tenemos en las sociedades, en lo que atañe, por ejemplo, a cubrir necesidades, alcanzar sueños y aportar a la propia historia de nuestros pueblos, sin necesidad de representantes de ninguna índole en asuntos relacionados con aspiraciones y necesidades, como tampoco en aquello de cambiar el entorno o afrontarlo. Esto tiene que ver con la autonomía, pero no únicamente la del individuo, también la colectiva o grupal. Los seres humanos, sea individual o colectivamente, estamos para ser protagonistas en asuntos de salud, para intervenir en igualdad persona-persona, colectivo-colectivo, no para representar a nadie ni para actuar en nombre de nadie. 
Quienes nos acercamos a la bioética cuando terminaba el siglo XX fuimos aceptando que, en esencia, se trataba de un saber interdisciplinar apoyado en la argumentación y la discusión en perspectiva de tomar decisiones que, a su vez, permitiesen resolver conflictos, como se dijo atrás, en el ámbito de la atención y de la investigación en biomedicina. Claramente, era una ética aplicada, es decir una herramienta puesta a punto para alcanzar esos fines, en buena parte ocasionados por el desarrollo tecno-científico y su impacto en sociedades multiculturales, por tanto, multi-morales, es decir, con diferentes principios y valores o diferentes concepciones del bien y del mal. Se trata de aplicar, desde arriba y afuera (única manera de ser imparciales, objetivos o racionales, expertos en eso de "analizar los hechos tal como ellos son"), unos principios estimados universales que han servido tanto para enmarcar el conflicto como para darle respuesta. El comité de ética es considerado el escenario natural para la discusión entre ese grupo de individuos vistos a sí mismos, o asignados, como representantes de los diferentes intereses en conflicto. Este grupo de individuos, mediante el diálogo, la discusión, el ejercicio de argumentación, de dar y escuchar razones al interior de dicho escenario de democracia, son quienes darán recomendaciones para buscar salidas al conflicto en el área de la atención o se encargarán de aprobar o rechazar una propuesta de investigación biomédica. Dialogo y democracia representativa (o comisionada) apuntarían a la toma de la mejor, o la menos dañina, de las potenciales salidas al conflicto en ciernes.

En cuanto a toma de decisiones, dichas democracias representativas o comisionadas, al menos las de este Tercer Mundo, han terminado favoreciendo los grandes negocios en manos de grandes negociadores. Los destinos de la vida en el planeta están al cuidado de unos pocos hombres poderosos que finalmente dirigen los rumbos de todas las democracias actuales con muy contadas excepciones. En el fondo, el valor de la vida para estos grandes negociadores tiene que ver con posibilidades para nuevos negocios. Son ellos quienes mantienen, ponen o quitan gobernantes, recomiendan o imponen modelos sanitarios y educativos, se apropian de los servicios públicos, desvían ríos, arrasan con bosques, desplazan poblaciones, etc., a todo lo largo y ancho del planeta. Es esta democracia representativa, comisionada o liberal,

la única legitimada y aceptada, siempre y cuando no comience a atentar contra los intereses hegemónicos occidentales. Si las poblaciones no-europeas no aceptan los términos de la democracia liberal, entonces se les impone por la fuerza, en nombre del progreso y la civilización" (GROSFOGUEL, 2007, p.74).

Al interior de democracias como estas hay muy poco respeto por el diálogo abierto y plural en perspectiva de buscar las mejores, o las menos malas

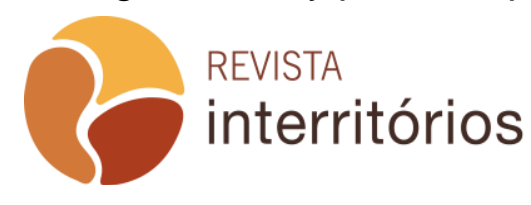


decisiones. Parodiando a Noam Chomsky en El miedo a la democracia (1992), se sostiene que somos libres de hacer lo que queramos, eso sí, siempre y cuando sea lo que se quiera que hagamos! Lo que se quiere que hagamos puede resumirse en vender y/o comprar, es decir, comerciar. Vivimos una sociedad que ha llevado a universalizar la mirada de que cada uno de nosotros es empresario de sí mismo. Hemos terminado construyendo una civilización mercantil, para la cual todo o casi todo vale si apunta a fortalecer las transacciones económicas, no importa si para fortalecerlas hay que reducir la democracia misma a extremos donde los gobernantes, independientemente de su tendencia política, están maniatados pues son las famosas manos e hilos invisibles del mercado, esencialmente financiero, sostenido por los más grandes poderes económicos del planeta, las que gobiernan, y son los mismos hilos y manos que evalúan lo bueno o malo de un gobierno, según como vaya a las mercancías y su flujo durante determinado mandato, según como vaya a las finanzas privadas. Al interior de un comité habrá voces y argumentos más fuertes o sólidos y finalmente serán esas voces y argumentos los que inclinarán la balanza de la toma de decisiones. Los comités al interior de nuestras sociedades pocos chances tienen, si alguno, de resultar más abiertas y plurales que las democracias representativas en que se ha asentado el ejercicio de la política en nuestros países.

\section{Pobreza, Movimientos Sociales y Bioética}

Aunque se acepta que algún papel de importancia jugaron los movimientos sociales por derechos civiles en los Estados Unidos de Norteamérica en el amplio contexto en que nació la bioética oficialmente con Van Rensselaer Potter, las diferentes perspectivas de bioética que han venido surgiendo luego de la versión finalmente hoy más difundida en el mundo (centrada en los cuatro principios: Autonomía, Beneficencia, No-maleficencia y justicia) nada volvieron a mencionar acerca de la relación entre bioética y movimientos sociales. Refiriéndose a las ideas en bioética, Giovanni Berlinguer anotaba.

Es difícil evaluar cuánto hayan pesado en los cambios de estas ideas, dos fenómenos concomitantes y convergentes: el progreso del conocimiento científico y la afirmación de procesos revolucionarios en política y cultura. Hablo de las luchas sociales y más aún, de los movimientos anticoloniales y antirracistas que surgen en los primeros dos tercios de nuestro siglo y del surgimiento de la cuestión femenina' en los últimos decenios. (BERLINGUER, 1993, p. 43). 
Esto que refería Berlinguer hace alrededor de 27 años, no ha dejado de ocurrir, ni los movimientos anticoloniales y antirracistas, ni la "cuestión femenina", se han detenido, mucho menos en Latinoamérica, donde los asesinatos de indios, negros y mujeres son el "pan de cada día". La razón para asesinarlos es simple: son principalmente estos grupos humanos quienes están poniendo en cuestionamiento precisamente la visión hegemónica de democracia, de bienestar, de progreso, de desarrollo, etc. Pareciera, eso sí, que las bioéticas han hecho a un lado estos asuntos. No ha podido saberse qué tanto y específicamente de qué manera los movimientos sociales aportaron al nacimiento de la bioética, pero al menos se reconoció en algún momento que algo tuvieron que ver en ese parto. Es más difícil entender por qué los bioeticistas dejamos de interesarnos en estos asuntos, sobre todo a este lado del mundo, Latinoamérica, donde "los nuevos conflictos culturales se oponen a las lógicas de concentración de poder que genera la tecno-economía de la información y los modelos de consumo y degradación ambiental que esta supone." (CALDERÓN, 2012, p.43).

Poco tiempo después de la conquista española (y portuguesa) en Latinoamérica surgieron movimientos indígenas y de negritudes, para quienes

la mejor ayuda que podrían prestarnos es dejarnos solos. La imposición o el paternalismo, el ataque o las campañas para incorporarnos a su cultura, solo sirven para continuar el proceso de nuestra destrucción. Tenemos una forma de vivir, de pensar, de morir, y necesitamos que esa cultura se nos respete. (SOTO, 1988, p. 26).

La pobreza finalmente es la mayor responsable de la vulnerabilidad social. De entre todos los seres humanos, los más pobres siguen siendo los más vulnerables y vulnerados. "No es exagerado decir que los pobres en América Latina están sufriendo un genocidio, de tal intensidad y extensión como no se conocía desde la Colonia." (ZIBECHI, 2017, p.10). Entre las diferentes perspectivas de bioética, prácticamente solo la Bioética de Intervención (BI) apunta explícitamente a la protección de estos vulnerables sociales, la fracción más débil de la sociedad. Es principalmente a causa de la pobreza que han surgido movimientos sociales que, en solitario, tratan de defender de la embestida privatizadora, empresarial o corporativa, lo poco que va quedando del bien común. Son movimientos sociales con características diferentes a los norteamericanos por derechos civiles de los años 60 y 70 del siglo XX, pues mientras el énfasis de aquellos estaba puesto en la inclusión, no sucede lo mismo en los movimientos contra-hegemónicos de esta parte del continente. Los movimientos sociales latinoamericanos, como algunos más en otros continentes, no persiguen la inclusión. 
Llamo la atención acerca del papel que están jugando los movimientos sociales contra-hegemónicos en el denominado Tercer Mundo, pero sobre todo en América Latina, que ponen en evidencia los límites de la discusión y de la democracia representativa cuando se abordan otros problemas claramente bioéticos, desde luego, bajo una mirada más amplia de la bioética centrada en el mero escenario de la atención y la investigación biomédicas.

Estos movimientos muestran precisamente ese protagonismo de los seres humanos que menciona la definición de salud expuesta unos cuantos párrafos atrás. Propongo que ahí se están caminando perspectivas de democracia y de diálogo que no precisamente acuden a comisiones o a representantes de nada ni de nadie, y que abordan asuntos relacionados con la vida en el planeta, todo esto, evidentemente de interés para la bioética.

Lo que surge en estos movimientos sociales lo hace remando contracorriente, no es una ética aplicada, desde arriba, desde una experticia o la combinación de varias, dirigida a dar respuesta(s) a problemas específicos acudiendo a unos principios pretendidamente universales, arropada en la comodidad de la distancia, en la tranquilidad que da no estar involucrados directamente, amparada en cierta supuesta imparcialidad.

Estos movimientos sociales latinoamericanos están andando una bioética eminentemente práctica, más cercana a lo que históricamente hemos hecho desde la conquista: resistir porque en la resistencia ha estado presente, y sigue estándolo, la re-existencia (BERNARDINO-COSTA, 2015) como pueblos en el mundo y también como individuos. La re-existencia es la puesta en marcha de una autonomía colectiva, no nada más individual, autonomía imprescindible para la sobrevivencia de indios y negros, hombres y mujeres de estas "razas inferiores", hoy percibidos por los poderosos como enemigos del progreso.

Ante un panorama como este, una propuesta intercultural de la bioética no parece caber en los espacios reducidos de los comités de asistencia o de investigación, quizá tampoco en las llamadas comisiones nacionales de bioética, enmarcados todos ellos en aquel ejercicio comisionado de democracia sustentado en el único tipo de conocimiento valido: el que aporta la ciencia y/o la racionalidad moderna. Se trata de buscar respuestas a los más grandes problemas relacionados con la vida en el planeta y que, en buena parte, se abordan bajo una única manera de entender la economía, de diseñar y aplicar tecnologías, de aprender y hacer ciencia, en fin, de conocer. Esa manera lleva más de 500 años siendo blanca, patriarcal, racional y europeo-norteamericana (GROSFOGUEL 2003, QUIJANO 2005) y ha cerrado el espacio por completo a otras epistemologías. Salud, ambiente y negocios son inseparables hoy en día. Bioética, ética ambiental y ética de los negocios, en la realidad cotidiana tampoco están separadas. Los grandes problemas que amenazan la vida en el planeta tienen que ver con estos asuntos y las éticas aplicadas siguen siendo éticas que 
acuden a esa única manera de entenderlos, una manera finalmente académica y experta, asentada en la colonialidad del poder y del saber. La interculturalidad no tiene posibilidades cuando una cultura se considera por encima de todas las demás.

En la costa pacífica colombiana, los pobladores, afrodescendientes en su mayoría, vieron aumentada su pobreza histórica a raíz del capital, el desarrollo y la guerra en la región, principalmente en los años 80. Por su parte, esa pobreza, impulsó la acción colectiva de resistencia, la cual ha venido consolidándose (ESCOBAR 2007, ZIBECHI 2019). Hoy su lucha puede enmarcarse en un concepto de biodiversidad bastante más complejo que la visión experta de la biología. La vida, el territorio y la cultura, todo ello junto, son su visión de biodiversidad y constituyen un mismo cuerpo, no pueden entenderla por separado. Esta perspectiva, desde luego, no es académica, sin embargo, es claramente bioética siempre y cuando veamos la bioética más ampliamente, una que ponga el énfasis en la vida, no solamente en la salud y mucho menos en los conflictos individuales de la atención y la investigación biomédicas. Esta perspectiva, de suyo, entra en perfecta consonancia con las raíces Bios y Ethos de la bioética, que también son inseparables. La vida (Bios) es territorio y es cultura (Ethos), ella es y está en el territorio, tanto como es y está en la cultura. Cultura y territorio son, bajo su perspectiva, vida en todas sus formas, cultura y territorio son, sin embargo, un Ethos localizado, no un Ethos abstracto o universal.

Movimientos sociales como este ponen en evidencia que ellos no solamente se ocupan de la injusticia distributiva, así este sea un problema relevante. Díaz y Luengo (2016), señalan que se ha dado una metamorfosis en los movimientos sociales, los cuales han pasado de la lucha por derechos a la lucha por la vida, de la formalidad jerárquica a la construcción de redes, de la relación con el Estado al autogobierno, de lo sectorial a la identidad cultural, de la homogeneidad a la heterogeneidad. En ese mismo sentido está su perspectiva de dignidad la cual no se concentra en la persona y sus derechos como individuo sino en el colectivo y sus necesidades de autodeterminación. No son solamente movimientos que reclaman ser escuchados y tenidos en cuenta en la toma de decisiones, sino que lo hacen mostrando en la práctica cotidiana que son posibles otras formas de relación con el otro ser humano, con el otro ser vivo, con la tierra o el cosmos, que hay otras formas de intercambio diferentes de aquel que exclusivamente persigue la ganancia. A su interior la teoría y la praxis avanzan (¡o retroceden!) juntas y sin demasiadas disertaciones acerca de qué debe ir primero. Moldiz (2013, p. 131) ha señalado que:

[...], en la teoría y práctica emancipadora estamos obligados a ampliar nuestros conceptos y uno de ellos es pasar del concepto reduccionista de desarrollo - que sólo hace referencia a cambio para el ser humano - al 
concepto amplio de vida - que es pensar en la especie humana y la naturaleza.

Hay una práctica bioética no experta al interior de movimientos sociales contra-hegemónicos en diferentes lugares de Nuestra América y en general del Sur global. Esa práctica bioética precisamente hace referencia a ese concepto amplio de vida. Aquel movimiento hindú por la protección de las semillas nativas (Navdanya) ante la embestida de las transgénicas y su móvil esencialmente comercial, agrupa desde agricultores analfabetos hasta brillantes profesionales e investigadores con maestrías y doctorados (SHIVA, 2006). Su idea de proteger las semillas naturales y autóctonas mediante un "banco" que por cada kilo de semillas que "presta" a los agricultores, estos le "devuelven" dos kilos al final de la cosecha, está protegiendo la biodiversidad en esa perspectiva de cuerpo como vida, territorio y cultura, a un mismo tiempo. No sólo muestra al mundo otras maneras de proteger el ambiente y a la vez garantizar la buena nutrición de las personas y los pueblos, un asunto de justicia, sino que investigan alrededor de sus propias necesidades, ejerciendo su derecho a una autonomía del colectivo, es decir, buscando el beneficio común más que individual. Es un movimiento que también muestra otras maneras de comercio, es decir, de mercado entre poblaciones y culturas, para las cuales el fin último no es la rentabilidad. No sólo plantea otras miradas, sino que adelanta acciones que ponen en evidencia la viabilidad de sus propuestas. En ellos confluye la deliberación interdisciplinar e intercultural con la acción social y política, por tanto, hay un ejercicio de democracia real, es decir activa y participativa, que permite tanto afrontar como transformar la sociedad, no simplemente adaptarse. Estos movimientos sociales anti-hegemónicos también son un refugio, un Ethos que ha permitido resistir a quienes históricamente fueron excluidos en las democracias representativas, a la vez que les permiten existir de maneras diferentes, es decir, re-existir.

Todo parece demostrar que la democracia comisionada no es todo lo pluralista y abierta a la deliberación que ostenta y que los movimientos sociales de finales del siglo XX y comienzos del XXI no son ataques de los malos y desadaptados a los buenos y su buena forma de vida en occidente. Es precisamente en estas democracias occidentales donde están ocurriendo.

¿Quién y cómo surgen los considerados representantes (hombres y mujeres) al interior de un movimiento social como este de los Sin Tierra en el Brasil, o Navdanya en la India, que también avanzan en otras partes? No hay respuesta a esta cuestión por la sencilla razón de que no son representantes de nadie, porque no hay comités aunque a su interior, en ocasiones y cuando los afanes cotidianos lo permiten, haya ejercicio de diálogo abierto y plural en la búsqueda de soluciones a variados problemas y se creen grupos que adelantan acciones específicas tendientes a mantener el movimiento, a conseguir apoyos, a protegerlo cuando menos de la manipulación mediática en manos de los más 
grandes intereses financieros del globo, entre otras. La guardia indígena de los Nasa en el departamento del Cauca (Colombia) es un ejemplo de esto. La necesidad de protegerse de la embestida de los diferentes actores violentos les impulsó a su creación. Todos los adultos, hombres y mujeres, en permanente rotación deben ser guardias durante algún período. Mientras lo son, el cuidado de sus familias y sus cultivos, corre a cargo de quienes no se ocupan de la guardia ese tiempo.

No podremos sostener que no hay democracia en estos movimientos, porque la hay y funciona, aunque de manera diferente por no requerir "representantes". Muestran otras formas de diálogo abierto y plural sin recurrir a comités. A su interior circulan diferentes formas de conocimiento y de ideas, pero también surge nuevo conocimiento y nuevas ideas al calor de sus luchas. Se trata de un conocimiento que no se aprende en las universidades, porque en ellas parece seguirse buscando verdades universales o susceptibles de universalidad, no importa si les dicen ciencias duras como la física o la biología, blandas como sicología o pedagogía. Se trata de un conocimiento sin pretensiones de objetividad porque cada persona influye en el movimiento y sus acciones, cómo éste y estas la influencian. Cada quien aporta tanto lo que conoce como lo que ignora y, finalmente nadie sabe quién tiene la verdad universal porque, de existir, sigue escondida en alguna parte, está en todos los lugares y en ninguno en particular. Se muestra, en fin, que aquello del conocimiento no es únicamente un asunto de racionalidad y que quizá lo sea, sobretodo, de relacionalidad. La racionalidad andina se basa en la relacionalidad entre todo lo que existe y el conjunto de sus experiencias vivenciales, las que son transmitidas de generación en generación (Blacutt, 2013, p.1).

Al interior de estos movimientos, aunque a veces se den discursos, nadie se toma la vocería por nadie, menos por todos, precisamente porque no hay representantes. Cuando surgen, los discursos buscan motivar a quienes se sienten derrotados o llamar la atención de gobiernos prepotentes que tienen todas las respuestas sin siquiera detenerse a pensar en las preguntas. Al interior de esos movimientos, es más probable que la ética se encuentre con la política porque los límites entre lo individual y lo colectivo son borrosos, ni caben sectarismos porque no hay quienes manden y quienes obedezcan. Ahí, sin duda, hay desorden. Sin embargo, qué más da, el orden en el mundo lo ha puesto completamente de cabeza y ha acabado por completo con la libertad al tornarnos a todos, o casi todos, esclavos de las mercancías.

La democracia occidental, dominada por la cultura del negocio, ha llevado a acabar con lo público, lo único que quedaba a los pobres, el lado históricamente más frágil de la sociedad (GARRAFA, 2005) y esa misma democracia occidental ha impulsado la autogestión de lo público por parte de los movimientos sociales en que se han convertido esas acciones colectivas en muchas partes del Sur global. A su interior se resuelven los asuntos de

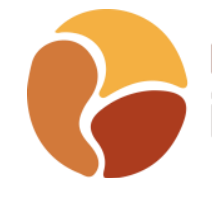


educación, salud, tierra, agua, alimento, vivienda, trabajo, etc., que la democracia les negó o les arrebató. Movimientos que finalmente, como han mostrado los Zapatistas en México, no están buscando el poder sobre el otro o los otros, persiguen simplemente un poder hacer conjuntamente, autónomamente, lo que estas democracias siempre impidieron (GÓMEZMULLER, 2014).

\section{Consideraciones Finales}

A finales de la década de los 90, en Brasil, se propone la Bioética de Intervención (BI) que "hace una alianza concreta con el lado históricamente más frágil de la sociedad” (GARRAFA, 2005, p.130), enfatiza esa alianza de tinte claramente ético-político y apunta a la intervención de los histórico-socialmente excluidos en la toma de decisiones. Sin embargo, sobre todo desde finales del siglo pasado y lo que ha trascurrido del presente, algunas de estas poblaciones se están moviendo por rumbos en que la inclusión no es su meta puesto que incluirse en esa visión rentable de la vida no les interesa, la ponen en cuestión y la confrontan. Eso, desde luego, les hace aún más frágiles porque enfrentan intereses supremamente poderosos tanto en lo económico, como en lo político y lo militar.

Si quienes nos inclinamos por la BI nos acercamos a movimientos sociales como éste en pos de la protección de las semillas nativas en la India (Navdanya), o el de los Sin Tierra en Brasil, o el de las comunidades indígenas y negras del pacífico y el sur en Colombia, podremos apreciar de primera mano formas distintas de democracia, lo cual, de alguna manera influirá en nuestra perspectiva futura para la Bl y en el modo de ejercerla, por cuanto los comités son espacios demasiado chicos para la puesta en marcha de todo su potencial hacia la protección de los vulnerables sean humanos o no, y hacia su intervención ante las injusticias o el daño. Estos movimientos abren las puertas para que los bioeticistas salgamos del encierro, no simplemente para que entren representantes de las diferentes visiones a unos comités que siguen siendo expertos y siguen quedándose cortos a la hora de encontrar respuestas a grandes dilemas que la técnica, el conocimiento y la ciencia plantean a sociedades, evidentemente, multi-morales.

No es un bioeticista (experto) quien va a hacer que el vulnerable (lego) se empodere, el asunto aquel del empoderamiento es activo, y precisamente expertos y legos al interior de movimientos sociales contra-hegemónicos van haciendo suyas teorías y prácticas emancipadoras. El ejercicio de emanciparse es cotidiano al interior de estos movimientos sociales, no importa su movil y si lo que persiguen nos gusta o no a diferentes tipos de expertos. Emancipación es también protección e intervención. Se requiere de intervención para proteger 
tanto como de protección para intervenir. El proceso de paz que apenas camina y está a punto de ser destruido en Colombia, muestra a las claras esta necesidad, si no hay protección no habrá posibilidades de intervención política de quienes dejan las armas, y si no hay intervención, la protección de los "históricamente" más frágiles seguirá postergada.

Las democracias occidentales siguen permitiendo la privatización del bien común. Creo que "seamos conscientes o no, la bioética constituye un lugar político donde la sociedad es confrontada con su propio devenir" (Durand, 1999, p125). Para negros en la costa pacífica colombiana, e indígenas en el Cauca, se trata de proteger "lo nuestro". En su visión, "Lo nuestro" es aquello que ni es tuyo ni es mío, como el agua, como la tierra, como el conocimiento, por eso tú y yo debemos cuidarlo, pero, además, ni tú ni yo podemos apropiárnoslo, si eso sucede, tú o yo tenemos el deber de que vuelva a ser nuestro. Proteger e intervenir están implícitos en esta visión "bárbara" de lo nuestro, el bien común.

Salud, ambiente y negocios no son campos independientes de la política y tampoco de la ética. Es el bien común de la humanidad lo que más se vulnera por parte de los grandes intereses financieros. Es ese bien común algo que se debería proteger de la privatización y que requiere de intervención cuando se arrebata por intereses individuales y privados. Por eso, nuestra $\mathrm{BI}$ no puede quedarse exclusivamente deliberando alrededor de los asuntos de la salud pública, sin hacerlo para los asuntos de la educación pública, de las semillas o los cultivos, del agua en manantiales y ríos, bienes comunes éstos que están siendo apropiados por grandes negociantes y puestos al servicio del lucro. Deberíamos contemplar la protección y la intervención a una escala mayor, el bien común no es solamente humano, es la vida en todas sus formas.

Como bioeticistas, promovemos la deliberación y la participación de las diferentes visiones en la toma de decisiones que de alguna manera a todos nos afectan, pero deberemos salir de los comités - al menos no quedarnos sólo ahí - porque en ellos no caben todas las miradas y hay otras formas de democracia que no son la comisionada o representativa la cual, aunque algo hace y permite hacer, muy poco podrá seguir aportando, hecho que cotidianamente también nos lo recuerdan esos movimientos sociales que se hacen sentir por estos tiempos a todo lo largo y ancho de las democracias occidentales.

Rara forma de democracia ésta, cada vez más parecida al totalitarismo que supuestamente contrarrestaría: el patentable por encima de cualquiera otro tipo de conocimiento, la comercial por encima de cualquiera otra manera de relación entre los seres humanos, el aseguramiento como única manera de promover la salud, prevenir la enfermedad, proteger, cuidar y tratar a los enfermos, la deliberación a puerta cerrada como única forma de exponer y escuchar argumentos. Si ese es el tipo de democracia que debe funcionar, no hay como detener el crecimiento y el surgimiento de un número cada vez mayor 
de movimientos sociales que la pongan en crisis, pero sobretodo con la ocurrencia innecesaria y evitable de muertes y sufrimiento, tanto de seres humanos como de otros seres vivos. De seguir por el mismo rumbo que hemos venido andando, quizá seamos cada vez más los bioeticistas que toquemos las puertas de los comités, esta vez no para entrar en ellos, más bien para salir.

\section{REFERENCIAS}

BERLINGUER, G. Questões de vida. Etica, ciência, saúde. APCE HUCITEC CEBES. Londrina, Brasil. 1993.

BERNARDINO-COSTA, J. Saberes subalternos e decolonialidade: Os sindicatos das trabalhadoras domésticas do Brasil. Editora UnB. Brasilia, Brasil. 2015.

BLACUTT-MENDOZA, M. El desarrollo local complementario. Un manual para la teoría en acción. Eumed,net/libros-gratis/2013/1252/1252.pdf

CALDERÓN, F. (Coordinadores). Cuadernos de Perspectiva Política. La protesta social en América Latina. PAPEP Perspectiva Política - PNUD - Siglo XXI Editores. Buenos Aires, Argentina. 2012.

CHOMSKY, N. El miedo a la democracia. Crítica. Barcelona, España. 2002. Diaz G, Luengo E. Los movimientos sociales: hacia otros mundos posibles. Ediciones desde abajo. Bogotá, Colombia. 2016.

DURAND, G. Introduction générale à la bioéthique. Histoire, concepts etoutils. FIDES.Cerf - Quebec, Canada. 1999.

GOMEZ-MULLER, A. Anarquismo: lo político y la antipolítica. Ediciones desde abajo, Bogotá, Colombia, 2014.

GARRAFA, V. Da bioética de princípios a uma bioética interventiva. Bioética (CFM), 13(1)125-134. 2005.

GARRAFA, V. \& OSORIO, L. Epistemología de la bioética - enfoque latinoamericano. $R B B, 3(3), 344-59.2007$.

GROSFOGUEL, R. Descolonizando los universales occidetales: El pluriversalismo transmoderno decolonial desde Aime Cesaire hasta los zapatistas. En El gito decolonia. Reflexiones para una diversidad epistemica mas alla del capitalismo global. Biblioteca Universitaria. Siglo del Hombre Editores. Bogota, Colombia, 2007.

KICKBUSCH, I. Promoción de la salud: una perspectiva mundial. En OPS/OMS (Ed.). Promoción de la salud: Una antología. Publicación científica 557 (15-24). Washington, USA, 1996.

POTTER, V. Global Bioethics: Building on the Leopold Legacy. Michigan State University Press. Michigan, USA, 1988. 
QUIJANO, A. Colonialidade do poder, eurocentrismo e América Latina. En: Lander E (Org). A colonialidades do saber: eurocentrismo e ciências sociais. Perspectivas latino-americanas. Consejo Latinoamericano de Ciencias Sociales. Buenos Aires, Argentina, 2005.

SOTO-APARICIO, F. Palabra de fuego. Plaza y Janés. Bogotá, Colombia, 1988.

SHIVA, V. Manifiesto para una democracia de la Tierra. Paidós. Barcelona, España, 2006.

WALSCH, C. Interculturalidad crítica y (de)colonialidad. Ensayos desde Abya Yala. Quito, Ecuador. 2012.

ZIBECHI, R. Movimientos sociales en América Latina. El "mundo otro" en movimiento. Ediciones Desde Abajo. Bogotá, Colombia, 2017.

ZIBECHI, R. Nuevas derechas, nuevas resistencias. Ediciones desde abajo. Bogotá, Colombia, 2019. 